PAPER

\title{
Impact of Noise on Spike Transmission through Serially Connected Electrical FitzHugh-Nagumo Circuits with Subthreshold and Suprathreshold Interconductances
}

\author{
Gonzalez-Carabarin Lizeth, Tetsuya Asai and Masato Motomura \\ Graduate School of Information Science and Technology, Hokkaido University \\ Kita 14, Nishi 9, Kita-ku, Sapporo 060-0814, Japan \\ E-mail: lizeth@1alsie.ist.hokudai.ac.jp, \{asai, motomura $\} @$ ist.hokudai.ac.jp
}

\begin{abstract}
This study is based on the utilization of noise as the main approach to overcoming the problems associated with spike transmission in neuromorphic excitable media, such as receiving weak signals as inputs. The main objective of this study is to consider the dependence of noise on membrane potential. This dependence allows proper spike transmission, discriminating weak signals from parasitic fluctuations that could generate a fake response. Inspired by this idea, we investigate how noise enhances the performance of spike transmission in serially connected electrical circuits receiving subthreshold inputs. Electrical simulation results for a small number of excitable circuits connected with each other showed that with the assistance of noise, spike transmission is activated even in the presence of subthreshold signals. Moreover, dynamical noise proved to be an efficient technique for preventing unwanted spikes.
\end{abstract}

Keywords: FitzHugh-Nagumo model, spike transmission, noise assistance

\section{Introduction}

It is known that under adverse conditions, such as the presence of noisy signals, many biological systems can complete some tasks efficiently. Then, the question that emerges is how this kind of system can overcome such conditions to perform these tasks. At the beginning of the ' $70 \mathrm{~s}$, in addition to traditional studies on the adverse effects of noise, many works were focused on the introduction of an optimal amount of noise to improve the system response. This phenomenon has been widely reported and is known as stochastic resonance (SR) [1]. As an example of noise-assisted tasks, we can find improvements in the ability to detect weak signals in biological systems [2]-[4]. Moreover, studies have demonstrated that the nervous system contains several sources of noise [5], and research in this field has demonstrated that the nervous system has adopted these noise sources to improve its efficiency. This kind of noise assistance can be observed in some arthropod mechanoreceptors [6], [7] as well as in the human sensory system and the brain [8], [9]. Following this background, in this study we focused on investigating the technique used by myelinated axons to transmit spikes [10]. This is in order to emulate their dynamics to assist weak signal propagation in transmission lines with high values of internodal resistors.

Transmitting correct information is a key factor in active transmission lines [11]. In this study, an active transmission line in a non-uniform medium is modeled as a chain of connected active compartments $A_{i}$, which are connected to each

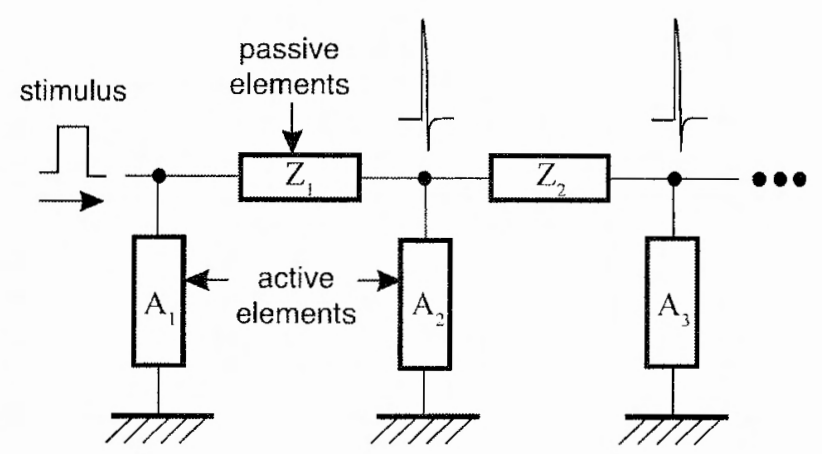

Fig. 1 Basic model of transmission line in nonuniform medium

other through internodal resistances $Z_{i}$ (Fig. 1). The values of the internodal resistances can fluctuate and sometimes acquire high values, causing loss of information. A similar electrical model of a transmission line is found in myelinated axons. Exploring how these biological structures are able to transmit information even in the presence of low conductances may help to improve information transmission in electrical systems. Furthermore, membrane-potential-dependent noise has been found in the nervous system that actually helps to transmit information [12]. This idea was used as the main approach in this study to distinguish low-amplitude inputs from fluctuations within the system.

This paper is organized as follows. In Sect. 2, we introduce 


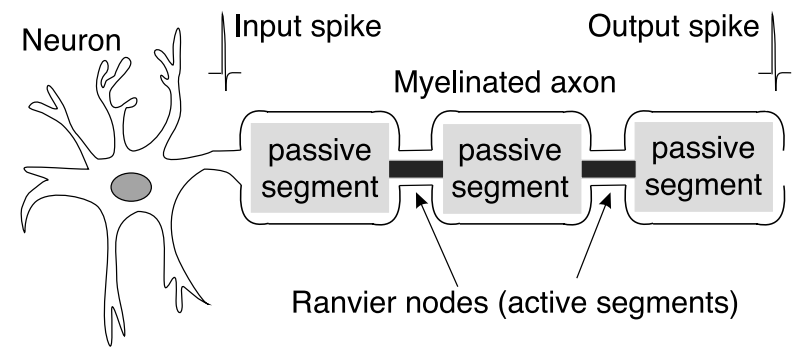

Fig. 2 General scheme of myelinated axons

our biological motivation. Section 3 presents the methodology of this study. In Sects. 4 and 5, we discuss the results of electrical simulations. Finally, the conclusion of the study is presented in Sect. 6.

\section{Biological Background}

Excitable systems are observed in a wide range of natural systems, and they can be defined as monostable systems. If such a system is left unperturbed, it will continue to rest; however, a strong perturbation can trigger the system to an excited state (called firing). In the nervous system, axons serve to transmit spikes in a neuronal excitable medium. Myelinated axons represent a very efficient biological technique for transmitting spikes along large axons. These kinds of axons are divided into segments that are separated from each other through high-density ion channel regions, known as Ranvier nodes (Fig. 2), which can be modeled electrically as a set of active components. On the other hand, low-density ion channel segments can be modeled as passive components. In this way, a myelinated axon is electrically represented as active elements connected to each other through passive segments. A fact about myelinated axons is that the internodal resistances associated with the low-density ion channel regions are not the same for every segment (mainly because of variations in dimensions along axons); thus, in the same axon, we can find segments that possess high resistivity as well as segments with low resistivity. In the case of high-resistivity segments, spike transmission will be terminated. However, it has been demonstrated that although high-resistance values exist, spike transmission is successful; thus, the question that arises is how such a biological design can transmit spikes successfully. The answer lies in the intrinsic noise generated by the opening and closing of ion channels inside the Ranvier nodes. This uncorrelated noise contributes to the improvement of the spike transmission rate. Information transmission with noise assistance has been studied extensively in bistable systems [13], [14] and electrical systems [15], [16].

However, previous studies have considered noise sources as independent sources. In this study, the effect of dynamical noise on the system response is introduced to improve signal propagation in excitable media. Experimental results from pyramidal neurons of rat neocortex show that intrinsic noise has a nonlinear dependence on membrane potential [17]. Steinmetz et al., using the conventional HodgkinHuxley model, predicted that the standard deviation of voltage noise increases as the membrane potential depolarizes, and the cause of this increment is the increase in the probability of ion channels opening [18]. In the following section, we further introduce the importance of including membranepotential-dependent noise for correct spike transmission in our current model.

\section{Electrical Model for Spike Transmission}

To emulate the Ranvier nodes as excitable compartments, we use the well-known FitzHugh-Nagumo model operating in an excitable mode. The FitzHugh-Nagumo model is a conductance-based model that has emerged as a simplified Hodgkin-Huxley model. The electrical FitzHugh-Nagumo model is defined by the following ordinary differential equations:

$$
\begin{aligned}
C \frac{d v}{d t} & =i-i_{\mathrm{d}}(v) \\
L \frac{d i}{d t} & =E-R \cdot i-v
\end{aligned}
$$

where $v$ is the membrane potential; $i$, the current; $E$, the resting potential; $R$, the load; and $i_{d}(v)$, the I-V characteristics of the tunnel diode [19].

On the other hand, if we consider a chain of electrical circuits connected to each other by resistors $R_{\mathrm{c}}$, the $j$ th circuit is described using the discrete FitzHugh-Nagumo equations as

$$
\begin{aligned}
C \frac{d v_{j}}{d t} & =i_{j}-i_{\mathrm{d}, j}+\frac{v_{j-1}+v_{j+1}-2 v_{j}}{R_{\mathrm{c}}} \\
L \frac{d i_{j}}{d t} & =E-R \cdot i_{j}-v_{j}
\end{aligned}
$$

where $R_{\mathrm{c}}$ represents the internodal resistance. Hence, the dynamics of a 1-D excitable medium (our virtual axon), where the excitable circuits are locally coupled, is represented by the following continuous forms:

$$
\begin{aligned}
C \frac{\partial v(x)}{\partial t} & =g \frac{\partial^{2} v(x)}{\partial x^{2}}+i(x)-i_{\mathrm{d}}[v(x)]+I_{\mathrm{n}}[v(x)](5) \\
L \frac{\partial i(x)}{\partial t} & =E-R \cdot i(x)-v(x)
\end{aligned}
$$

where $x$ denotes the space; $v(x)$, the membrane potential at $x$; $g$, the nominal internodal conductance; $i_{\mathrm{d}}[v(x)]$, the I-V characteristics of the tunnel diode; $E$, the resting potential; and $I_{\mathrm{n}}[v(x)]$, the $v(x)$-dependent dynamic noise current where 


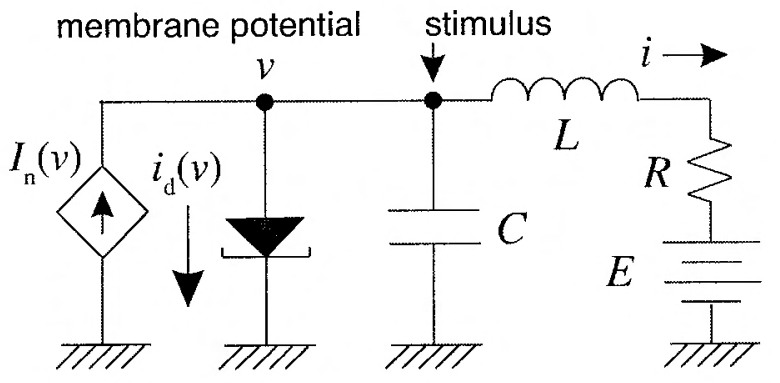

Fig. 3 Noise-driven FitzHugh-Nagumo circuit

the noise current is generated only when $v>E$. The hypothesis of $I_{\mathrm{n}}[v(x)]$ is qualitatively consistent with the experimental and simulation results shown in [17] and [18], where the standard deviation of noise increases as the membrane depolarizes. The activation of noise sources only when $v>E$ was adopted because it represents the simplest case of the dependence of noise on membrane potential, which ensures the activation of noise sources only in the presence of subthreshold inputs, thereby discriminating weak inputs from internal fluctuations. Therefore, the characteristic of $I_{\mathrm{n}}[v(x)]$ is crucial for successive spike transmission; that is, if noise is generated independently of $v$, excitable circuits that should not be depolarized (receiving no input) may be depolarized by the noise, whereas if noise is generated only when $v>E$, the circuits may be depolarized only when inputs (external stimuli or firing of the neighbors) are given, even if they are below the threshold of the depolarization. Figure 3 represents the FitzHugh-Nagumo electrical circuit, wherein the membranepotential-dependent noise source is in parallel with the tunnel diode. Figure 4(a) defines the symbol of the $j$ th (conventional) FitzHugh-Nagumo circuit, whereas Fig. 4(b) shows $N$ serially connected FitzHugh-Nagumo circuits, with the membrane-potential-dependent noise sources, $I_{\mathrm{n}}\left(v_{j}\right)$, represented by Eqs. 3 and 4 .

\section{Simulations and Results}

SPICE simulations were conducted for this excitable system with the following parameters: tunnel diode NEC 1S1760, $C=0.1 \mathrm{nF}, R=0.2 \Omega, L=10 \mu \mathrm{H}$, and $E=50$ $\mathrm{mV}$. Nine excitable circuits $(j=1-9)$ were locally connected by resistors $R_{\mathrm{c}}\left(\sim g^{-1}\right)$, and the first circuit on the boundary was stimulated by an external current pulse (amplitude: $0.5 \mathrm{~mA}$, width: $1 \mu \mathrm{s}$ ). When $R_{\mathrm{c}}$ was $1 \mathrm{k} \Omega$, successive spike transmission was observed (from the first to the ninth circuit) without noise assistance, whereas spike transmission was randomly terminated when $R_{\mathrm{c}}$ was $1.5 \mathrm{k} \Omega$ also without noise assistance. As can be observed in Fig. 5, postsynaptic potential was not generated, and only a small perturbation was observed in the second circuit. This phenomenon occurs as a result of the reduction in the input spike due to the suprathresh-

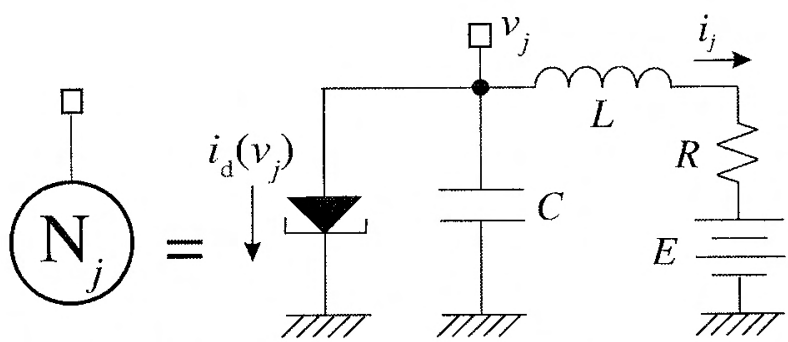

(a) Conventional FitzHugh-Nagumo circuit

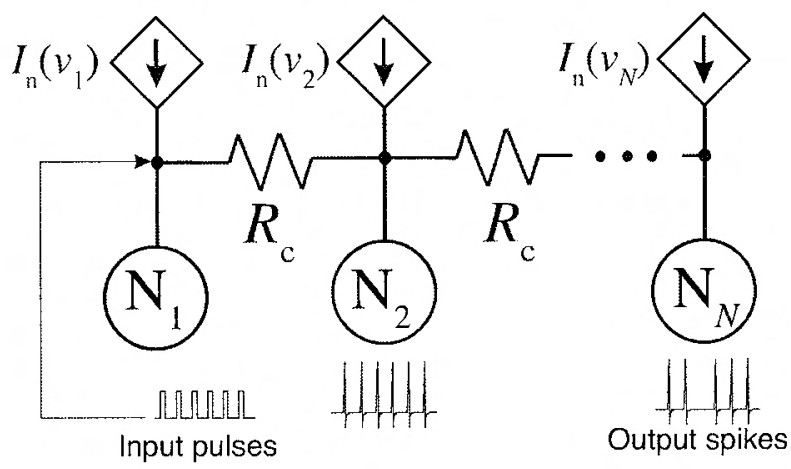

(b) Serially connected FitzHugh-Nagumo circuits with noise sources

Fig. 4 Circuit configuration

old value of the internodal resistances, and therefore, it is not possible to excite the subsequent stages, thus resulting in zero response. This figure clearly shows that spike transmission is terminated in the presence of suprathreshold values for internodal resistors.

On the other hand, when noise sources were introduced (additive white Gaussian noise with $\sigma=0.45 \mathrm{~mA}$ and $\mu=$ 0 ), spike transmission was successful. Figure 6 shows spike propagation for the $1 \mathrm{st}, 5$ th, 7 th, and 9 th circuits, showing that with the introduction of noise sources, spike propagation was achieved successfully. Figure 7 shows time courses of the membrane potentials of circuits $1,3,5$, and 9 , which are connected through suprathreshold values for internodal resistors. Periodic current pulses were applied to the 1 st circuit as an excitatory input (period: $10 \mu \mathrm{s}$ ). Because of the stochastic nature of the noise sources, spike transmission was not successful in some cases; therefore, in the following simulations, we introduce the spike transition rate as the percentage of spikes transmitted to each circuit.

It is necessary to explore how the standard deviation of noise affects the performance of spike transmission. Several simulations were carried out using different values of standard deviation. Figure 8 shows the spike transmission rate for circuits 3, 5, 7, and 9. Monte Carlo simulations were run with 100 iterations for 21 different values of standard deviation, and in Fig. 8, the continuous lines represent the average of 10 Monte Carlo simulations. As observed in Fig. 8, the 


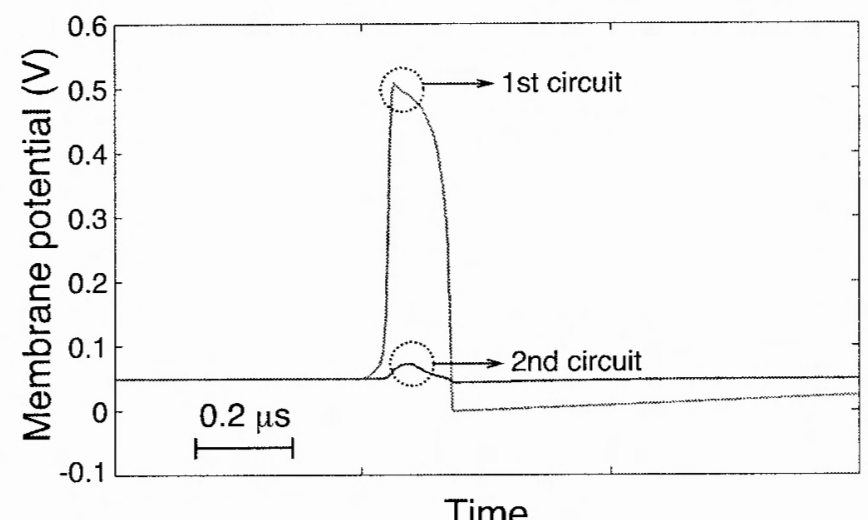

Fig. 5 Membrane potential in the 1 st and 2 nd circuits without noise assistance

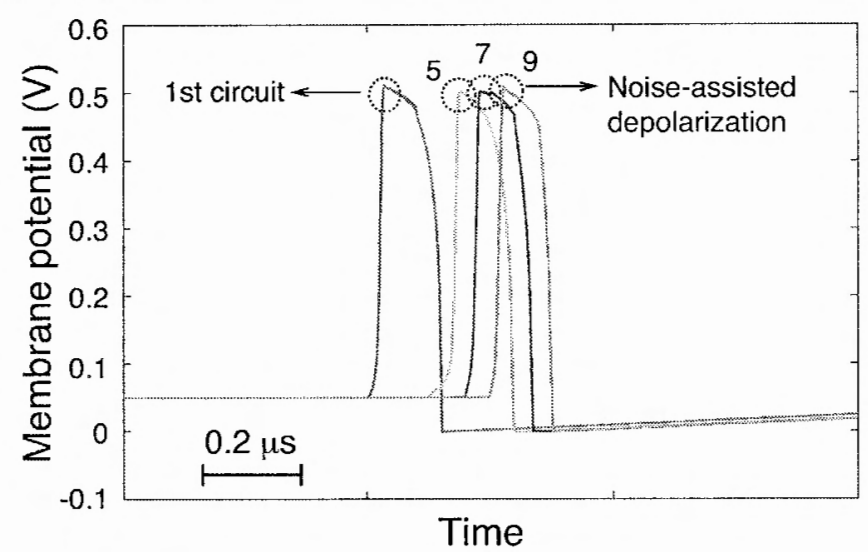

Fig. 6 Membrane potential in the 1st, 5 th, 7 th, and 9 th circuit with noise assistance

generation of a spike in the subsequent circuits becomes more probable as the standard deviation of noise increases. If the amplitude of the noise input is independent of the membrane potential, SR-like behavior can be observed. However, in this particular case of study, the noise is dependent on the membrane potential; therefore, weak noise signals may not be able to assist weak inputs in exciting the subsequent stages; however, as the amplitude of the noise signal increases, the probabilities of generating a spike also increase. Moreover, we found that there is a specific value at which the spike transmission rate stops increasing (around $0.8 \mathrm{~mA}$ ) and remains constant. However, even if SR-like behavior is not observed, the main advantage of the introduction of the noise-dependent current source is that unwanted spikes are prevented. If the amplitude of the noise signal is independent of the circuit parameters, then when the noise signal is high enough, it can rise to spike activity even in the absence of excitatory inputs.
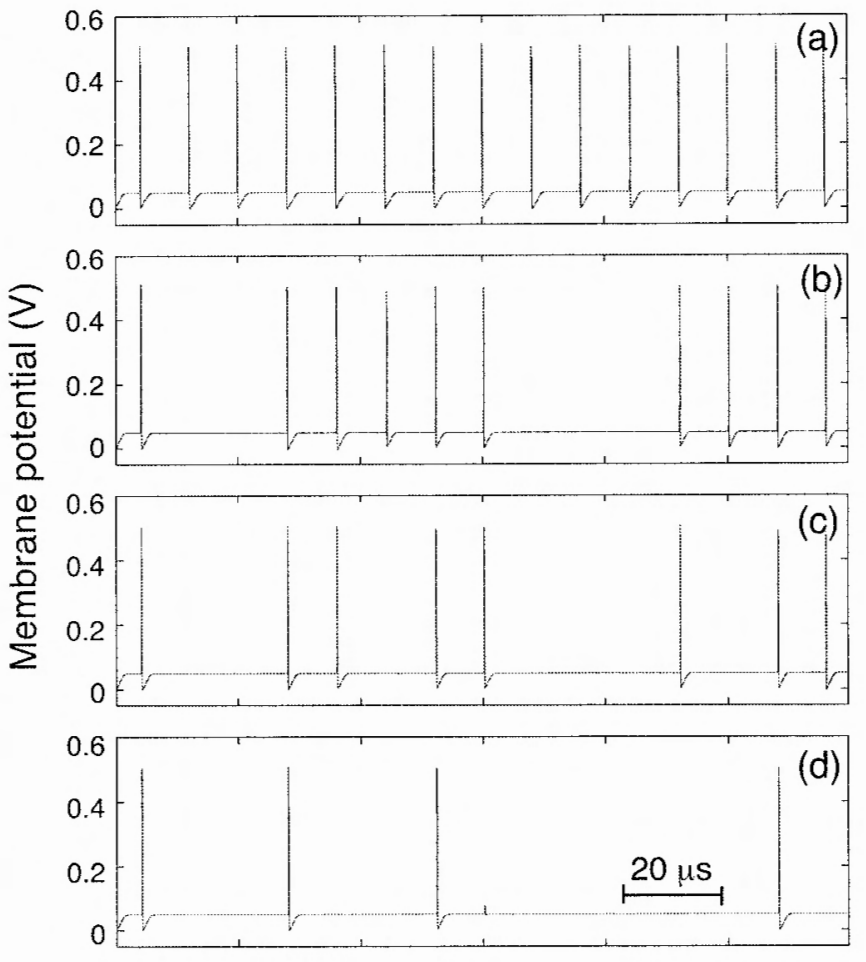

Time

Fig. 7 Spike trains of the (a) 1 st, (b) 3 rd, (c) 5 th, and (d) 9 th circuits assisted by noise

\section{SR-like Behavior from Subthreshold and Suprathreshold Internodal Resistances}

Thus far, we have investigated the effect of internal fluctuations with a view to improving the spike transmission rate along a transmission line, where internodal resistances were set to a specific value. This value must be large enough to induce a failure in the spike transmission. Thus far, this value has remained constant for all the iterations. In actual systems, this value fluctuates and has different values for each resistance. Therefore, it is necessary to expand this study to a more realistic case; thus, we move our attention to the study of random fluctuations of internodal resistors. We used uniformly distributed random numbers to assign values to the internodal resistances in the interval $\left(0, R_{\max }\right]$. A graph of the spike transmission rate versus $R_{\max }$ was expected to be represented by a decreasing function. However, simulation results with values of $R_{\max }$ from $10 \Omega$ to $3.5 \mathrm{k} \Omega$ demonstrated that there was a specific range of values for internodal resistances at which spike transmission reached its maximum rate (Fig. 9). The bell-shaped curve implies that the best performance is achicved only for a specific value of $R_{\max }$. For low $R_{\max }(10$ to $40 \Omega$ ), the spike transmission was completely unsuccessful because the membrane potentials at each node 


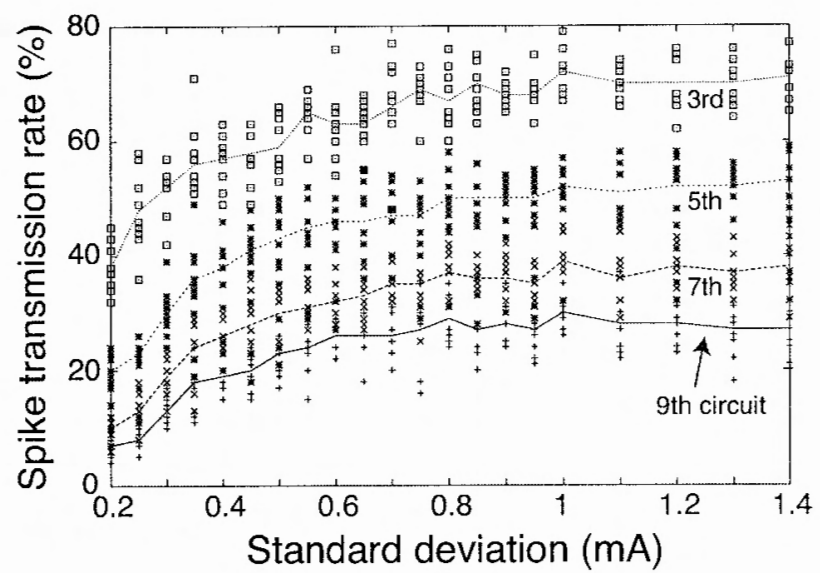

Fig. 8 Spike transmission rate at the $3 \mathrm{rd}, 5$ th, 7 th, and 9 th circuit versus standard deviation of noise

were strongly coupled by the small internodal resistances, and hence the nominal threshold values for firing were increased. These simulations show promising results since the network exhibits a new kind of SR-like behavior. Additional simulations were also run simultaneously for different values of the standard deviation of noise, as shown in Fig. 10. As the standard deviation increased, the right tails of undulations of $R_{\max }$ expanded, which clearly showed that the noise improved the spike transmission rate at higher internodal resistances.

\section{Conclusion}

The introduction of background noise to enhance the spike transmission rate for a population of biological neurons was explored. Motivated by this idea, we improved the weak signal transmission in serially connected circuits on the basis of the stochastic FitzHugh-Nagumo electrical circuit to develop noise-assisted active transmission lines consisting of coarsegrained devices and materials as a future objective. Simulation results showed that spike propagation was enhanced by applying current noise to the membrane potential node. We determined the value for subthreshold resistors where the spike transmission rate was maximum. When the value of internodal resistances started increasing until a suprathreshold value, without noise assistance, it was impossible to generate a spike at subsequent circuits; however, when noise was applied, a spike was more likely to be generated in the subsequent circuits, showing that even weak stimuli can excite the next circuit with noise assistance.

The key factor in this study that resulted in successful spike transmission was the introduction of a membrane-potentialdependent current source as the noise input. Since a noise source is only activated when there are subthreshold stimuli, parasitic spike generation is prevented. However, the effect

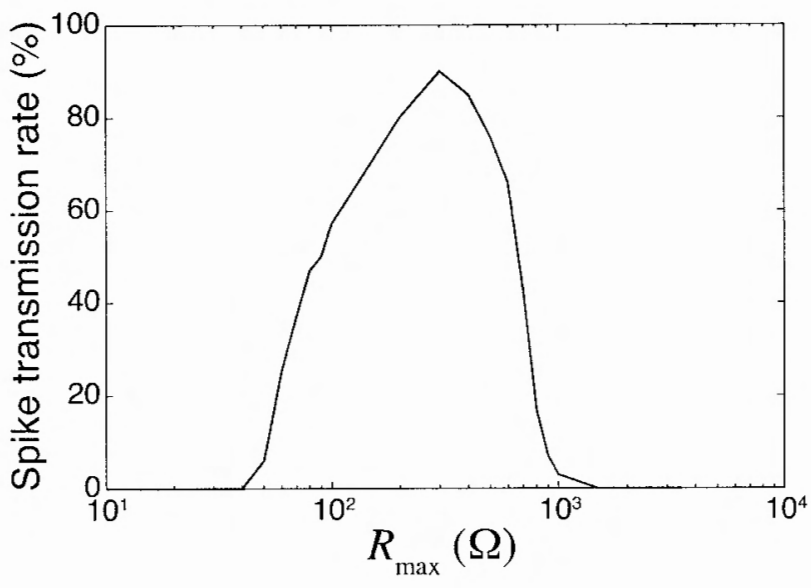

Fig. 9 Spike transmission rate versus maximum internodal resistance $R_{\max }$

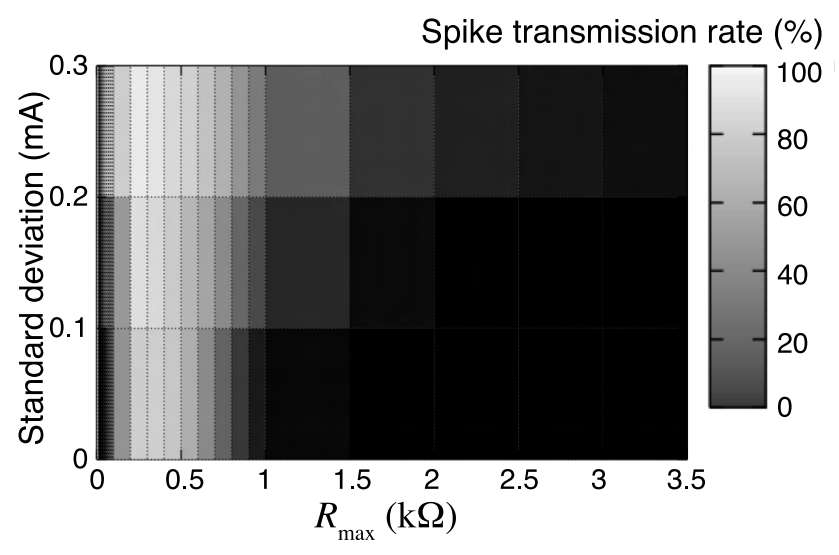

Fig. 10 Spike transmission rate versus maximum internodal resistance $R_{\max }$ and standard deviation of noise

of the standard deviation on the performance of spike propagation must be considered in detail, because there is a critical value for noise amplitude where the spike transmission rate stops increasing and fluctuates around a certain value. Although SR-like behavior was not exhibited, spike transmission was improved considerably by the assistance of noise. Moreover, we emphasized the results from the simulations by assigning random values for internodal resistances (subthreshold and suprathreshold cases), which represent the closest model to actual systems, and observed a new kind of SRlike behavior.

\section{Acknowledgements}

This study was supported by a Grant-in-Aid for Scientific Research on Innovative Areas [20111004] from the Ministry of Education, Culture, Sports, Science and Technology (MEXT) of Japan. 


\section{References}

[1] L. Gammaitoni, P. Hänggi, P. Jung and F. Marchesoni: Stochastic resonance, Rev. Mod. Phys., Vol. 70, No. 1, pp. 223-287, 1998.

[2] W. C. Stacey and D. M. Durand: Stochastic resonance improves signal detection in hippocampal CA1 neurons, J. Neurophysiol., Vol. 83, No. 3, pp. 1394-1402, 2000.

[3] H.-Y. Li, J. Ma, Z.-H. Hou and H.-W. Xin: Constructive role of internal noise for the detection of weak signal in cell system, Acta Phys.-Chim. Sin., Vol. 24, No. 12, pp. 2203-2206, 2008.

[4] M. Kawaguchi, H. Mino and D. M. Durand: Stochastic resonance can enhance information transmission in neural networks, IEEE Trans. Biomed. Eng., Vol. 58, No. 7, pp. 1950-1958, 2011.

[5] A. A. Faisal, L. P. J. Selen and D. M. Wolpert: Noise in the nervous system, Nat. Rev. Neurosci., Vol. 9, No. 4, pp. 292-303, 2008.

[6] J. K. Douglass, L. Wilkens, E. Pantazelou and F. Moss: Noise enhancement of information transfer in crayfish mechanoreceptors by stochastic resonance, Nature, Vol. 365, No. 6444, pp. 337-340, 1993.

[7] X. Pei, L. A. Wilkens and F. Moss: Light enhances hydrodynamic signaling in the multimodal caudal photoreceptor interneurons of the crayfish, J. Neurophysiol., Vol. 76, No. 5, pp. 3002-3011, 1996.

[8] J. J. Collins, T. T. Imhoff and P. J. Grigg: Noiseenhanced tactile sensation, Nature, Vol. 383, No. 6603, p. $770,1996$.

[9] K. Kitajo, D. Nozaki, L. M. Ward and Y. Yamamoto: Behavioral stochastic resonance within the human brain, Phys. Rev. Lett., Vol. 90, No. 21, pp. 218103(1)(4), 2003.

[10] A. Ochab-Marcinek, G. Schmid, I. Goychuk and P. Hanggi: Noise-assisted spike propagation in myelinated neurons, Phys. Rev. E, Vol. 79, No. 1, pp. 011904(1)(7), 2009.

[11] C. S. Lindquist: Active transmission lines-characteristic impedance and propagation functions, Proc. IEEE, Vol. 57, No. 8, p. 1422-1423, 1969.

[12] J. A. White, R. Klink, A. Alonso and A. R. Kay: Noise from voltage-gated ion channels may influence neuronal dynamics in the entorhinal cortex: J. Neurophysiol., Vol. 80, No. 1, pp. 262-269, 1998.
[13] J. F. Lindner, S. Cramouli, A. R. Bulsara, M. Locher and W. L. Ditto: Noise enhanced propagation: Phys. Rev. Lett., Vol. 81, No. 23, pp. 5048-5051, 1998.

[14] A. Zaikin, D. Topaj and J. Garcia-Ojalvo: Noiseenhanced propagation of bichromatic signals: Fluct. Noise Lett., Vol. 2, No. 1, pp. L47-L52, 2002.

[15] A. C. H. Rowe: Experimental observation of stochastic resonance in a linear electronic array, Phys. Rev., Vol. 64, pp. $031106(1)-(4), 2001$.

[16] S. Morfu, J.-C. Comte, J.-C. Bilbault and P. Marquié: Noise-enhanced propagation in a dissipative chain of triggers, Int. J. Bifurcat. Chaos, Vol. 12, No. 3, pp. 629 $633,2002$.

[17] G. A. Jacobson, K. Diba, A. Yaron-Jakoubovitch, Y. Oz, C. Koch, I. Segev and Y. Yarom: Subthreshold voltage noise of rat neocortical pyramidal neurones, J. Physiol., Vol. 564, No. 1, pp. 145-160, 2005.

[18] P. N. Steinmetz, A. Manwani, C. Koch, M. London and I. Segev: Subthreshold voltage noise due to channel fluctuations in active ncuronal membranes, J. Comput. Neurosci., Vol. 9, No. 2, pp. 133-148, 2000.

[19] J. Nagumo, S. Arimoto and S. Yoshizawa: An active pulse transmission line simulating nerve axon. Proc. IRE, Vol. 50, No. 10, pp. 2061-2070, 1962.

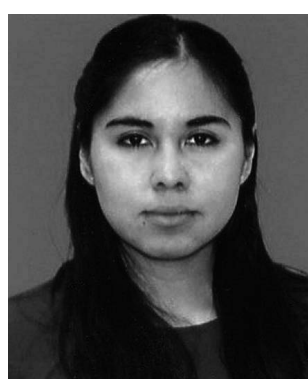

Gonzalez-Carabarin Lizeth received her B.S. degree in electronic engineering from the Technological Institute of Orizaba, Mexico, in 2007, and her M.S. degree from the Research and Advanced Studies Center of the National Polytechnic Institute, Mexico City, in 2009. Currently she is working towards her PhD degree in the Department of Electrical Engineering at Hokkaido University, Japan. Her research interests include neuromorphic circuits and stochastic resonance.

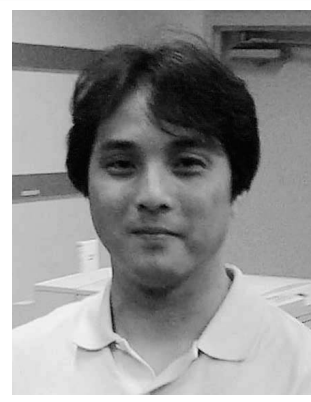

Tetsuya Asai received his B.S. and M.S. degrees in electronic engineering from the Tokai University, Japan, in 1993 and 1996, respectively, and his Ph.D. degree from the Toyohashi University of Technology, Japan, in 1999. He is now an Associate Professor in the Graduate School of Information Science and Technology, Hokkaido University, Sapporo, Japan. His research interests are focused on developing nature-inspired integrated circuits and their computational 
applications. Current topics that he is involved with include intelligent image sensors that incorporate biological visual systems or cellular automata in the chip, neurochips that implement neural elements (neurons, synapses, etc.) and neuromorphic networks, and reaction-diffusion chips that imitate vital chemical systems.

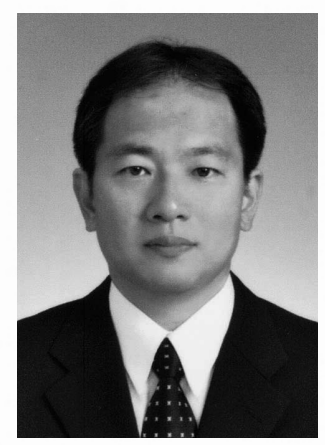

Masato Motomura received his B.S. and M.S. degrees in physics and his Ph.D. degree in electrical engineering in 1985, 1987, and 1996, respectively, all from Kyoto University. He was with NEC and NEC Electronics from 1987 to 2011 , where he was engaged in the research and business development of dynamically reconfigurable processors and on-chip multicore processor architectures. $\mathrm{He}$ moved to Hokkaido University in April 2011, where he is now a Professor in the Graduate School of Information Science and Technology. His current research interests include reconfigurable and parallel architectures and low-power circuits. He won the IEEE JSSC Annual Best Paper Award in 1992, the IPSJ Annual Best Paper Award in 1999, and the IEICE Achievement Award in 2011. He is a member of IEICE and IEEE.

(Received May 23, 2012; revised September 18, 2012) 